\title{
SOAP-V: Introducing a Method to Empower Medical Students to be Change Agents in Bending the Cost Curve
}

\author{
Eileen M. Moser, MD, MHPE, Grace C. Huang, MD²,3, Clifford D. Packer, MD4, Susan Glod, MD¹, Cynthia D. Smith, MD5,
} Patrick C. Alguire, MD5, Sara B. Fazio, $\mathrm{MD}^{3}$

\begin{abstract}
${ }^{1}$ Department of Medicine, Milton S. Hershey Medical Center, Penn State College of Medicine, Hershey, Pennsylvania; '2Shapiro Institute for Education and Research, Harvard Medical School, Beth Israel Deaconess Medical Center, Boston, Massachusetts; ${ }^{3}$ Department of Medicine, Harvard Medical School, Beth Israel Deaconess Medical Center, Boston, Massachusetts; ' 4 Department of Medicine, Case Western Reserve University School of Medicine, Cleveland, Ohio; ${ }^{5}$ American College of Physicians, Philadelphia, Pennsylvania.
\end{abstract}

Medical students must learn how to practice high-value, cost-conscious care. By modifying the traditional SOAP (Subjective-Objective-Assessment-Plan) presentation to include a discussion of value (SOAP-V), we developed a cognitive forcing function designed to promote discussion of high-value, cost-conscious care during patient delivery. The SOAP-V model prompts the student to consider (1) the evidence that supports a test or treatment, (2) the patient's preferences and values, and (3) the financial cost of a test or treatment compared to alternatives. Students report their findings to their teams during patient care rounds. This tool has been successfully used at 3 medical schools. Preliminary results find that students who have been trained in SOAP-V feel more empowered to address the economic healthcare crisis, are more comfortable in initiating discussions about value, and are more likely to consider potential costs to the healthcare system. Journal of Hospital Medicine 2016;11:217-220. (C) 2015 Society of Hospital Medicine
Today's medical students will enter practice over the next decade and inherit the escalating costs of the US healthcare system. Approximately $30 \%$ of healthcare costs, or $\$ 750$ billion dollars annually, are spent on unnecessary tests or procedures. ${ }^{1}$ High healthcare costs combined with calls to eliminate waste, improve patient safety, and increase quality ${ }^{2}$ are driving our healthcare system to evolve from a fee-based system to a value-based system. Additionally, many patients are being harmed by overtesting and the stress associated with rising healthcare bills. Financial risk has increasingly shifted to patients in the form of higher deductibles and reduced caps, and medical indebtedness is the number 1 risk for bankruptcy. ${ }^{3,4}$ False positive results of low-yield diagnostic tests lead to additional testing, anxiety, excess radiation exposure, and unnecessary invasive procedures. ${ }^{5}$ To minimize harm to patients, evidence must guide physicians in their ordering behavior. In addition, any care plan a physician develops should be individualized to incorporate patients' values and preferences. Unfortunately, medical students, who are at an impressionable stage in their careers, frequently observe overtesting and unnecessary treatment behaviors in their clinical encounters. ${ }^{6}$ Instead, our medical students and train-

\footnotetext{
*Address for correspondence and reprint requests: Eileen Moser, MD, Penn State College of Medicine, Office of Medical Education, Mail Code H176, 500 University Drive, Hershey, PA 17033; Telephone: 717-5310003; Fax: 717-531-3925; E-mail: emoser1@hmc.psu.edu

Additional Supporting Information may be found in the online version of this article.

Received: June 6, 2015; Revised: August 17, 2015; Accepted: August 26, 2015

2015 Society of Hospital Medicine DOI 10.1002/jhm.2489

Published online in Wiley Online Library (Wileyonlinelibrary.com).
}

ees must be prepared to deliver patient care that is evidence based, patient centered, and cost conscious. They must become effective stewards of limited healthcare resources.

To help prepare our students for this evolving healthcare paradigm, we created a new tool called SOAP-V (Subjective-Objective-Assessment-Plan-Value), designed to embed discussion of healthcare value into medical student oral presentations and note writing. Students are encouraged to use this tool at the point of care to bring up value concepts with physicians and residents as part of medical decision making. In so doing, we propose that medical students can serve as change agents to shift physician practice at our academic medical centers to focus on healthcare value. This article describes the SOAP-V tool, contains links to educational materials to help hospitalists and other clinician educators to implement this tool, and provides preliminary findings and reflections.

\section{INNOVATION}

SOAP-V was conceived at the Millennium Conference on Teaching High-Value Care, which was sponsored by the Beth Israel Deaconess Medical Center Shapiro Institute for Education and Research, the Association of American Medical Colleges, and the American College of Physicians. Educators from several medical schools decided to form a group to specifically consider ways to train medical students and residents in the concept of high-value care (HVC), which is framed as improving patient outcomes while decreasing patient cost and harm. ${ }^{7}$ Our group recognized several challenges in teaching HVC. First, physician practice habits are influenced by the way they are trained, ${ }^{8}$ yet faculty who teach those future physicians 
frequently have not themselves been taught, nor do they consistently practice HVC. ${ }^{9}$ Second, we needed to teach students the requisite HVC knowledge, attitudes, and skills, and therefore wanted to provide opportunities to not only learn, but practice, HVC, preferably in authentic patient experiences to optimize their learning. ${ }^{10,11}$ Third, we recognized that adding another teaching task to the already oversubscribed day of an attending might understandably be met with resistance. We envisioned a tool that could be used with minimal or no faculty training, could be attached to authentic patient experiences, and based on LEAN-Six Sigma principles, ${ }^{12}$ would be embedded in the normal workflow. Furthermore, we considered social networking principles, such as those described by Christakis and Fowler that describe how an individual's behavior impacts behaviors of those surrounding them, ${ }^{13}$ and hoped to empower medical students to serve as change agents. Medical students could initiate discussions of value concepts at the point of care in a way that challenges a heavily entrenched test-ordering culture and encourages other members of the team to balance potential benefit with harms and cost. Following the conference, the group held bimonthly phone conferences and subsequently developed the SOAP-V tool, created teaching materials, and planned a research project on SOAP-V.

SOAP-V modifies the traditional SOAP (SubjectiveObjective-Assessment-Plan) oral presentation or medical note, to include value (V). It serves as a cognitive forcing function designed to create a pause and promote discussions of HVC during patient delivery. It prompts the student to ask 3 value questions: (1) Before choosing an intervention, have I considered whether the result would change management? (2) Have I incorporated the patient's goals and values, and considered the potential harm of the intervention compared to alternatives? (3) What is the known and potential cost of the intervention, both immediate and downstream? The student gathers information during the patient interview and brings back the information to the team during rounds where management decisions are made.

In the summer of 2014, we launched an institutional review board-approved, multi-institutional study to implement SOAP-V at Penn State College of Medicine, Harvard Medical School, and Case Western Reserve University School of Medicine for third-year medical students during their internal medicine clerkships. Students in the intervention arm participated in an interactive workshop on SOAP-V. Authors S.F., S.G., and C.D.P., who serve as clerkship directors in internal medicine, provided student training for each cohort of intervention students at the beginning of each rotation on general medicine inpatient wards. The workshop began with trigger videos that demonstrate pressures encountered by a student on rounds that might lead to overuse. ${ }^{14}$ Following a discussion on overuse and methods to avoid overuse, the students were introduced to the SOAP-V framework, watched a video of a student modeling a SOAP-V presentation on rounds, ${ }^{15}$ and engaged in a SOAP-V role play. They received a SOAP-V pocket card as well as a Web link to Healthcare Bluebook ${ }^{16}$ to research costs. An outline of the session and associated materials can be found in an online attachment. ${ }^{17}$ The students then used the SOAP-V tool during inpatient rounds. We advised supervising faculty that students might present using a SOAP-V format, and provided them with a SOAP-V card, but we did not provide faculty development on SOAP-V. Students participating in the control arm did not receive training specific to SOAP-V.

Students in intervention and control arms at each school were surveyed on their attitudes toward HVC at the beginning of the clerkship year and then again at the completion of the medicine clerkship via a 19item questionnaire soliciting perceptions and selfreported practices in HVC. Intervention arm students received biweekly e-mail links that allowed them to anonymously document their use of SOAP-V, as well as an end-of-clerkship open-ended question about the usefulness of SOAP-V. We analyzed questionnaire results using McNemar's test for paired data.

\section{PRELIMINARY FINDINGS}

The preintervention attitudinal survey $(\mathrm{n}=226)$ demonstrated that although $90 \%$ of medical students agreed on the importance of considering costs of treatments, only $50 \%$ felt comfortable bringing up cost considerations with their team, and $50 \%$ considered costs to the healthcare system in clinical decisions. An interim analysis of the available data at 6 months (response rate approximately $50 \%$ across sites) showed that students in the intervention arm reported increased agreement with the phrases, "I have the power to address the economic healthcare crisis" (pre$37 \%$, post $-65 \%, P=0.046$ ); "I would be comfortable initiating a discussion about unnecessary tests or treatments with my team," (pre- $46 \%$, post $-85 \%, P=$ 0.027); and "In my clinical decisions, I consider the potential costs to the healthcare system" (pre-41\%, post- $60 \%, P=0.023$ ) compared to control arm students, who showed no significant differences pre- versus postrotation in these 3 domains (Figure 1).

To date, biweekly surveys and direct observation of rounds have verified student use of SOAP-V. Student comments have included: "Allowed me the ability to raise important issues with the team while feeling like I was helping my patients and the healthcare system." "A great principle that I used almost daily." "Great to implement this at such a "young" stage in my med career." "Broadened my perspective on the role of a physician."

SOAP-V has inspired some of our medical students to consider value in healthcare more closely. In a 


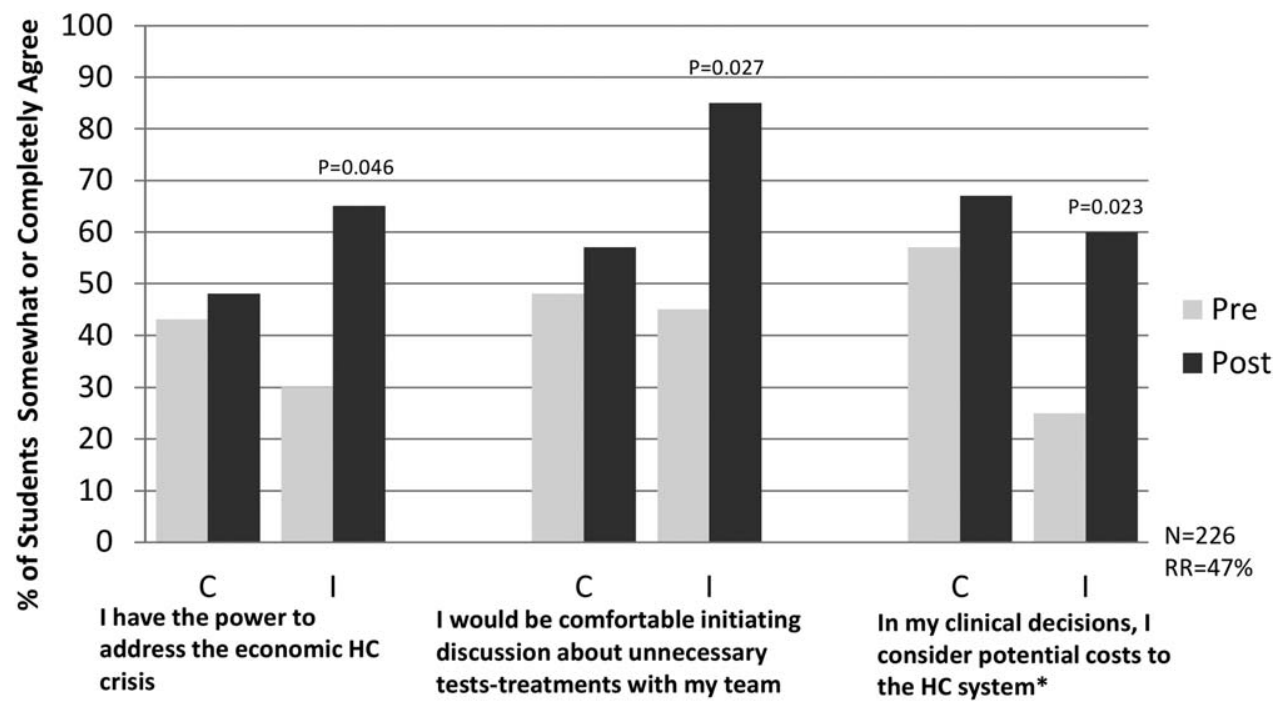

FIG. 1. Third-year students from 3 medical schools $(n=226)$ participated in a survey on their attitudes on high-value care immediately prior to the start of third year and following completion of their internal medicine clerkship. Six-month interim data (response rate $=47 \%$ ) of student agreement with statements pre- versus postintervention are presented. ${ }^{*}$ The difference between the control and intervention group in this question was not statistically significant $(P=0.06)$. Abbreviations: $\mathrm{C}$, control group; $\mathrm{HC}$, healthcare; I, intervention group; $\mathrm{RR}$, relative risk.

notable example, a SOAP-V-trained student admitted a young man with lymphadenopathy, pulmonary infiltrates, and weight loss who underwent an extensive and costly workup including liver biopsy, bronchoscopy, and multiple computed tomography and positron emission tomography scans and was eventually diagnosed with sarcoidosis. The SOAP-V-trained student reviewed the patient's workup, estimated that the team spent more than $\$ 6000$ to make the diagnosis, and recommended a more cost-effective approach.

Common barriers experienced by the pilot sites included time constraints limiting discussion of value, variability in perceived receptivity depending on team leadership, and student confidence in initiating this dialogue. Solutions included underscoring the notion that value discussions can be brief, may be appropriately initiated by any member of the team, and may have an effect on choice of management and/or patient preference issues that can make medical care more efficient and effective. Resident and faculty physicians were made aware of the intervention, and encouraged to support students in using the SOAP-V tool.

\section{CONCLUSION}

SOAP-V was successfully implemented within the inpatient internal medicine clerkship at 3 academic institutions. Our preliminary results demonstrate that students can use this framework to apply considerations of high-value, cost-conscious care in their medical decision making and to promote discussion of these concepts during rounds with their inpatient teams. Students in the intervention arm report greater comfort discussing unnecessary tests and treatments with their team and a greater likelihood to consider potential costs to the healthcare system. Additionally, these students commented that the SOAP-V framework broadened their perspective on their role as a physician in curbing costs, and that they felt more empowered to address the economic healthcare crisis. The next phase of our project will involve conducting end-of-year surveys to evaluate whether SOAP-V has a persistent impact on the frequency and quality of value discussions on rounds, as well as students' attitudes about cost consciousness. We will also gauge whether resident and faculty attitudes about HVC have changed as a result of the intervention.

Our SOAP-V student training was provided in a 1hour session. We believe that the ease of training and the simplicity of the SOAP-V framework permit SOAP-V to be easily transferred for use by residents, medical students in other clerkships, and other healthcare learners. Additional research is needed to demonstrate this expanded use and prove sustainability. An additional important question is whether use of SOAP-V by students and residents results in reductions in unnecessary costs. Future educational efforts will include embedding the SOAP-V tool in other clerkships and promoting the SOAP-V tool within corresponding residencies in both hospital and outpatient clinic settings and analyzing potential reductions in wasteful spending.

It is generally conceived that medical students learn the information they are taught, and are impacted by the culture in which they reside; multiple studies bear this out. ${ }^{18,19}$ However, students may also be change agents. Our students will inherit the healthcare systems of the future. We must empower them to change the status quo. There can be tremendous utility in employing such a "bottom up" approach to process improvement. What a student discusses today may spark the resident (or faculty) to consider in their own 
workflow tomorrow. In this way, we envision that the SOAP-V is a tool by which ideas concerning HVC can be generated and shared at the point of care. It is our hope that this straightforward intervention is one that may slowly change the culture and perhaps eventually the practice patterns of our academic medical centers.

Disclosure: Nothing to report.

\section{References}

1. Institute of Medicine. The Healthcare Imperative: Lowering Costs and Improving Outcomes. Washington, DC: The National Academies Press; 2010.

2. Institute for Healthcare Improvement. IHI triple aim initiative. Available at: http://www.ihi.org/Engage/Initiatives/TripleAim/pages/default. aspx. Accessed August 7, 2015.

3. Himmelstein DU, Thorne D, Warren E, Woolhandler S. Medical bankruptcy in the United States, 2007. Am J Med. 2009;122(8):741746.

4. The Henry J. Kaiser Family Foundation. Health care costs: a primer. Key information on health care costs and their impact. May 2012. Available at: https://kaiserfamilyfoundation.files.wordpress.com/ 2013/01/7670-03.pdf. Accessed August 7, 2015.

5. Greenberg J, Green JB. Over-testing: why more is not better. Am J Med. 2014;127:362-363.

6. Tartaglia KM, Kman N, Ledford C. Medical student perceptions of cost-conscious care in an internal medicine clerkship: a thematic analysis [published online May 1, 2015]. J Gen Intern Med. doi: 10.1007/ s11606-015-3324-4.

7. Owens DK, Qaseem A, Chou R, Shekelle P. High-value, costconscious health care: concepts for clinicians to evaluate the benefits, harms, and costs of medical interventions. Ann Intern Med. 2011;154: 174-180.

8. Weinberger SE. Providing high-value, cost-conscious care: a critical seventh general competency for physicians. Ann Intern Med. 2011; 155:386-388.

9. Korenstein D, Kale M, Levinson W. Teaching value in academic environments: shifting the ivory tower. JAMA. 2013;310(16):16711672.

10. Knowles MS, Holton EF, Swanson RA. Theories of teaching. In: The Adult Learner. New York, NY: Routledge; 2012:72-114.

11. Hodges B. Medical education and the maintenance of incompetence. Med Teach. 2006;28:690-696.

12. De Koning H, Verver JP, van den Heuvel J, Bisgaard S, Does RJ. Lean Six Sigma in healthcare. J Healthcare Oual. 2006;2:4-11

13. Christakis NA, Fowler JH. Connected. New York, NY: Little, Brown \& Company; 2009.

14. Teaching Value Project. Costs of care. Available at: teachingvalue.org Available at: https://www.dropbox.com/s/tb8ysfjtzklwd8g/OverrunPart1.webm; https://www.dropbox.com/s/cxt9mvabj4re4g9/OverrunPart2.webm. Accessed August 7, 2015

15. Moser EM, Fazio S, Huang G. SOAP-V [online video]. Available at: https://www.youtube.com/watch?v=goUgAzLuTzY\&feature=youtu. be. Accessed August 7, 2015.

16. Moser EM, Fazio S, Huang G, Packer C, Glod S. SOAP-V workshop and materials. Available at: https://drive.google.com/folderview? id=0B99pzr6gQcbefnRqVktNN21 mTC0tTWctYmxRSVd3ajFsOEZ odWFWNzUzdXQwa2RUcmpHa28\&usp=sharing. Accessed August 7, 2015.

17. Healthcare Bluebook. Available at: https:/HealthcareBluebook.com. Accessed July 25, 2015.

18. Mossop L, Dennick R, Hammond R, Robbe I. Analysing the hidden curriculum: use of a cultural web. Med Educ. 2013;47(2):134-143.

19. Karani R, Fromme HB, Cayea D, Muller D, Schwartz A, Harris IB. How medical students learn from residents in the workplace: a qualitative study. Acad Med. 2014:89(3):490-496. 